\title{
Dual Langmuir-Probe Array for 3D Plasma Studies in TORPEX
}

\author{
M. Baquero-Ruiz, ${ }^{1}$ F. Avino, ${ }^{1}$ O. Chellai, ${ }^{1}$ A. Fasoli, ${ }^{1}$ I. Furno, ${ }^{1}$ R. Jacquier ${ }^{1}$ F. Manke, ${ }^{1}$ and $S$. Patrick ${ }^{1}$ \\ Swiss Plasma Center (SPC), Ecole Polytechnique Fédérale de Lausanne (EPFL), CH-1015 Lausanne, \\ Switzerland
}

(Dated: 4 November 2016)

We have designed and installed a new Langmuir-probe (LP) array diagnostic to determine basic 3D features of plasmas in TORPEX. The diagnostic consists of two identical LP arrays, placed on opposite sides of the apparatus, which provide comprehensive coverage of the poloidal cross-section at the two different toroidal locations. Cross-correlation studies of signals from the arrays provide a basic way to extract 3D information from the plasmas, as experiments show. Moreover, the remarkable signal-to-noise performance of the frontend electronics allows us to follow a different approach in which we combine information from all probes in both arrays to reconstruct elementary 3D plasma structures at each acquisition time step. Then, through data analysis, we track the structures as they evolve in time. The LP arrays include a linear-motion mechanism that can displace radially the probes located on the Low Field Side for experiments that require fine-tuning of the probe locations, and for operational compatibility with the recently-installed in-vessel Toroidal Conductor.

\section{INTRODUCTION}

Langmuir-probes (LPs) are some of the most widely used diagnostics in experimental plasma physics ${ }^{1}$. They consist of a small metallic tip that is inserted into a plasma which, upon measurement of the current-voltage (I-V) characteristic, makes it possible to deduce the local plasma density $(n)$, plasma potential $\left(V_{p}\right)$ and electron temperature $\left(T_{e}\right)$.

Provided the tip can tolerate the plasma environment ${ }^{2}$, LPs can be used for measurements even in the plasma bulk. This is routinely done in basic plasma physics experiments like TORPEX (Sec. II), where the low plasma densities and temperatures allow LP access in essentially all the interior of the apparatus. In fact, several LPs can be inserted at the same time to perform measurements simultaneously at different locations. TORPEX ${ }^{3-5}$ as well as other experiments with toroidal ${ }^{6}$ or linear geometries $^{7,8}$, have used two-dimensional (2D) arrays of LPs to observe and study the evolution of plasma structures in 2D sections of the devices. These studies have significantly contributed to the understanding of perpendicular dynamics (across magnetic field lines) of plasma blobs $^{9}$ of importance for fusion grade devices. LP arrays have also enabled experimental studies of parallel dynamics (along the magnetic field), leading to important results in, among others, wavenumbers of fluctuations ${ }^{10}$.

There are still, however, many open questions on the experimental three-dimensional (3D) dynamics of blobs. Furthermore, 3D effects are suspected to be at the origin of many differences currently observed between experimental data and models of blobs ${ }^{9}$. Indeed, many theories neglect variations along the magnetic field lines, an assumption that is well motivated in most cases but may not be appropriate in general ${ }^{9}$. It can happen, for example, that parallel variations trigger instabilities ${ }^{11}$. Observations of blobs that include 3D information are therefore important to expand the understanding of blob dynamics and to help develop more complete theoretical models.
In this paper, we describe the design, calibration and implementation of the HEXagonal Turbulence Imaging Probe Upgrade (HEXTIP-U), a new diagnostic consisting of two LP arrays installed on different toroidal locations that allows us to determine basic 3D features of TORPEX plasmas in different magnetic field configurations.

Fully exploiting the ability to change magnetic fields in TORPEX without sacrificing poloidal cross-section coverage is a challenging requirement that calls for an innovative array design (Sec. II). Comprehensive crosssection coverage also requires the use of a significant number of probes (190), each one with its own front-end electronics. A careful calibration and characterization of the electronics, as described in Sec. III, is therefore needed to assure data consistency across all channels (probes) and for a correct interpretation of front-end output signals.

The capabilities of the complete, calibrated, system are determined with experiments performed in TORPEX. The results, shown in Sec. IV, are followed by a discussion and an outlook of potential studies made possible by HEXTIP-U (Sec. V).

\section{TORPEX AND THE HEXTIP-U DIAGNOSTIC}

The TORoidal Plasma EXperiment (TORPEX) $)^{3,12}$ is a toroidal plasma device of major radius $R=1 \mathrm{~m}$ and minor radius $a=20 \mathrm{~cm}$ located at the Swiss Plasma Center (SPC) in Lausanne, Switzerland. To obtain a hydrogen plasma (other gases can also be used ${ }^{3}$ ), $\mathrm{H}_{2}$ gas is introduced in the experimental vessel (initially at a pressure $p \leq 2 \times 10^{-6}$ mbar) until reaching $p \approx 10^{-5}-10^{-4}$ mbar, and then microwaves of frequency $f_{\mathrm{mw}}=2.45 \mathrm{GHz}$ and power $P_{\mathrm{mw}} \leq 1.0 \mathrm{~kW}$ are injected to produce a discharge by absorption at the electron-cyclotron and upper-hybrid resonances ${ }^{13}$. In Simple Magnetized Torus (SMT) configurations, a small vertical magnetic field $B_{z} \lesssim 5 \mathrm{mT}$ is superposed on a dominant toroidal magnetic field $B_{\phi} \approx 70 \mathrm{mT}$. In that case we obtain hydrogen plasmas 


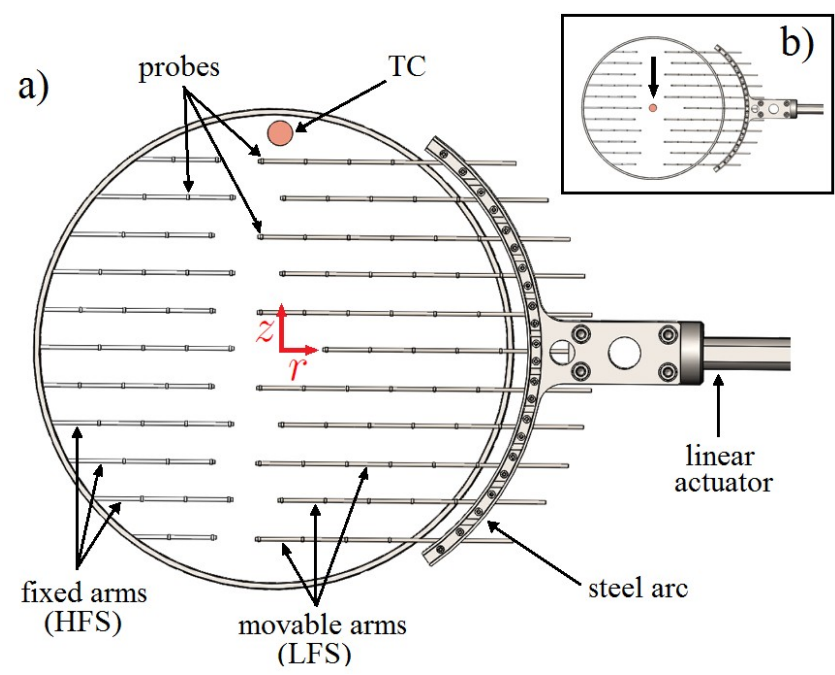

FIG. 1. Each of the two LP arrays of HEXTIP-U is made of a set of Low Field Side (LFS) probes resting on movable ceramic arms and a complementary set of fixed High Field Side (HFS) probes. (a) When the LFS arms are not displaced, the system forms an hexagonal grid which provides comprehensive LP coverage of the entire poloidal cross-section. Notice the definition of the coordinates $r, z$ (in red). (b) The LFS arms can be displaced radially (along $r$ ) to allow the TC to move unobstructed.

with typical densities $n \approx 10^{15}-10^{17} \mathrm{~m}^{-3}$, temperatures $T_{e} \approx 5 \mathrm{eV}$ and plasma potentials $V_{p} \approx 10-20 \mathrm{~V}$.

More complicated magnetic configurations have recently become possible with the use of the in-vessel copper Toroidal Conductor ${ }^{14,15}$ (TC). These include closed magnetic flux surfaces ${ }^{16}, \mathrm{X}$-points ${ }^{17}$ and magnetic snowflakes ${ }^{18}$, which require the TC to be set at different vertical positions in the vessel. This constraint conflicts with the installation of LP arrays because their delicate structure could be damaged during the displacement of the TC to new locations. Indeed, former LP arrays ${ }^{19}$ had to be uninstalled every time the TC needed adjustment. This sensitive operation could itself damage the diagnostics. HEXTIP-U solves this issue with a linear actuator that displaces part of the array so that a gap is opened in the region where the TC is expected to move (Fig. 1). The actuator can also be used to fine-tune the radial location of the Low Field Side (LFS) probes for studies that require a precise radial positioning of the probes (see Sec. IV).

HEXTIP-U comprises two identical LP arrays installed on opposite sides of the toroidal vessel. Each array is composed of 95 LPs made of stainless steel. The probes are hollow cylinders of inner diameter $3.05 \mathrm{~mm}$, thickness $0.5 \mathrm{~mm}$ and length $2.0 \mathrm{~mm}$. They are beaded on hollow cylindrical ceramic supports, or arms, of inner diameter $2.0 \mathrm{~mm}$, outer diameter $3.0 \mathrm{~mm}$ and length up to $269 \mathrm{~mm}$. Holes are laser-drilled into the ceramic at a constant spacing of $35 \mathrm{~mm}$ to provide a path through which teflon-insulated stainless-steel wires connect to the

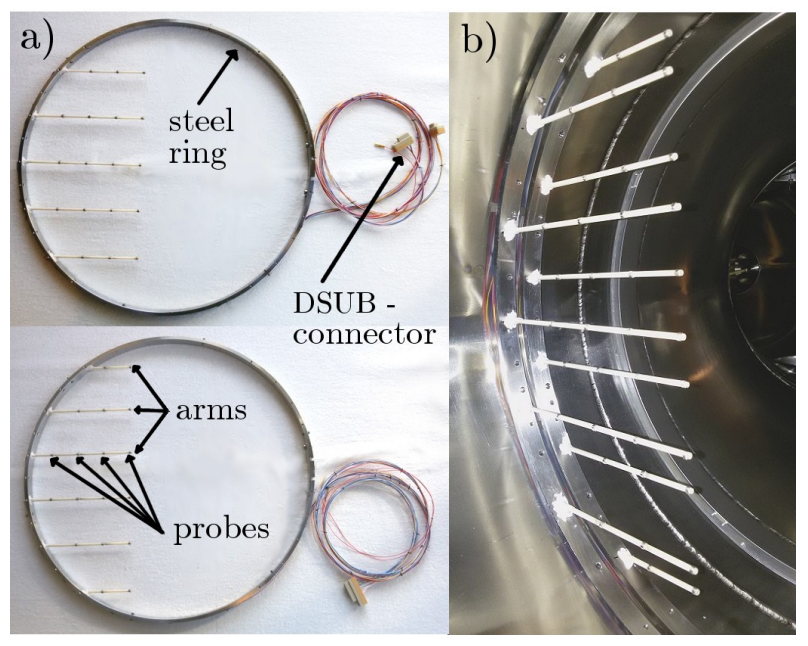

FIG. 2. HFS probes. Final assembly of two stainless-steel rings holding the ceramic arms with LPs (a) before and (b) after installation in the vacuum vessel.

probes by spot-welding. This in turn fixes the spacing between probes along the arms at $(35 \pm 1) \mathrm{mm}$.

The 95 probes are distributed on a total of 22 arms. Eleven of these arms are mounted on two stainless-steel rings of diameter $39 \mathrm{~cm}$ and glued using liquid-ceramics (Fig. 2a). This is similar to a setup used in earlier studies ${ }^{19}$. For installation, the rings (complete with arms and probes) are placed next to one another and secured to the inner wall using screws. Together, the two rings form a hexagonal 2D array of 36 LPs that covers most of the High Field Side (HFS) area of the poloidal cross section (Fig. 2b). Coverage is restricted to $r \leq-35 \mathrm{~mm}$, where $r$ is the radial poloidal coordinate (see Fig. 1), to provide enough space around $r=0$ to allow the TC to move unobstructed in the vertical direction when needed.

The remaining 59 probes rest on a different set of 11 arms. These are not fixed to rings but rather are secured to two stainless-steel arcs attached to a newly-designed in-vacuum linear-motion structure (Fig. 3). One arc holds five arms (27 probes) while the other holds six arms (32 probes) using a clamping mechanism that guarantees a good horizontal alignment. We estimate a vertical position uncertainty $\leq 2 \mathrm{~mm}$ (typically less) for all probes from measurements during assembly. Each full arc assembly can be moved in the radial direction, independently of the other, by up to $20 \mathrm{~mm}$ towards the HFS (negative $r$ ) or up to $70 \mathrm{~mm}$ towards the LFS (positive $r$ ). The precision is better than $1 \mathrm{~mm}$. When neither of the arcs is displaced, this setup provides complete coverage of the poloidal cross section with a hexagonal grid with lattice constant $35 \mathrm{~mm}$. Turning the linear-motion actuators allows displacing both arcs to, for example, open a gap between ceramic arms near $r=0$ for TC height adjustments (Fig. 1b). A differential pumping system guarantees safe operation of the actuators in normal experimental conditions when the vessel is under high vac- 

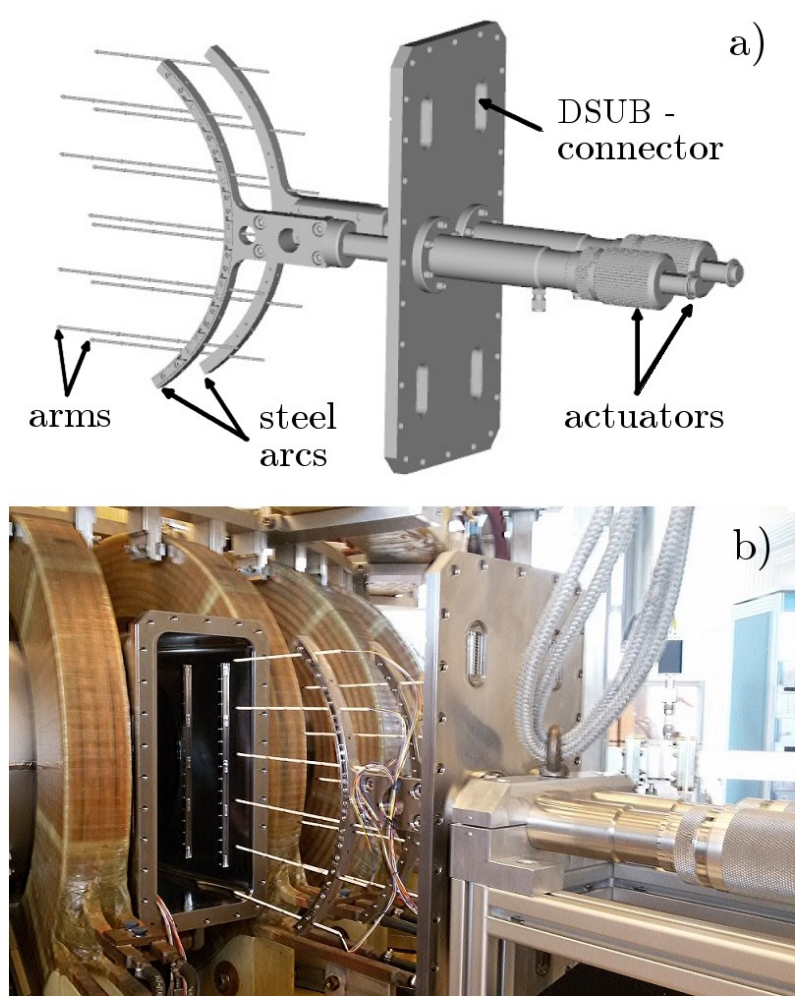

FIG. 3. LFS probe assembly. (a) Design schematic which shows the support arcs for the ceramic arms and the linear motion mechanism. (b) Complete LFS assembly during installation.

uum.

The monopole wires connecting to all probes are bundled and inserted into four 25-pole female DSUB connectors made of PEEK. Each connector is attached to one of four DSUB-25 male-to-male plugs welded onto the flange that supports the movable system (Fig. 3a). The flange, in turn, is installed and fixed in place with bolts on one of the wedge-shaped ports of the apparatus (Fig. 3b). This provides a vacuum-safe connection of the probes to the exterior of the vessel and to the front-end electronics through four shielded 25-pole cables.

The two identical copies of the full array assembly, with independent front-end electronics and data digitizers, are installed with a toroidal separation of $180^{\circ}$ (Fig. 4).

\section{ELECTRONICS AND DATA ACQUISITION}

Theoretical models make it possible to determine experimental values of $n, V_{p}$ and $T_{e}$ from measurements of the current-voltage (I-V) characteristics of Langmuirprobes $^{1,2,20}$. The behavior of LPs in the presence of magnetic fields is still not fully understood ${ }^{1,2}$, but several simplifying assumptions can be made that lead to useful interpretations of LP data. One such case is the magnetized quasi-collisionless model $^{2}$, in which ions are as-

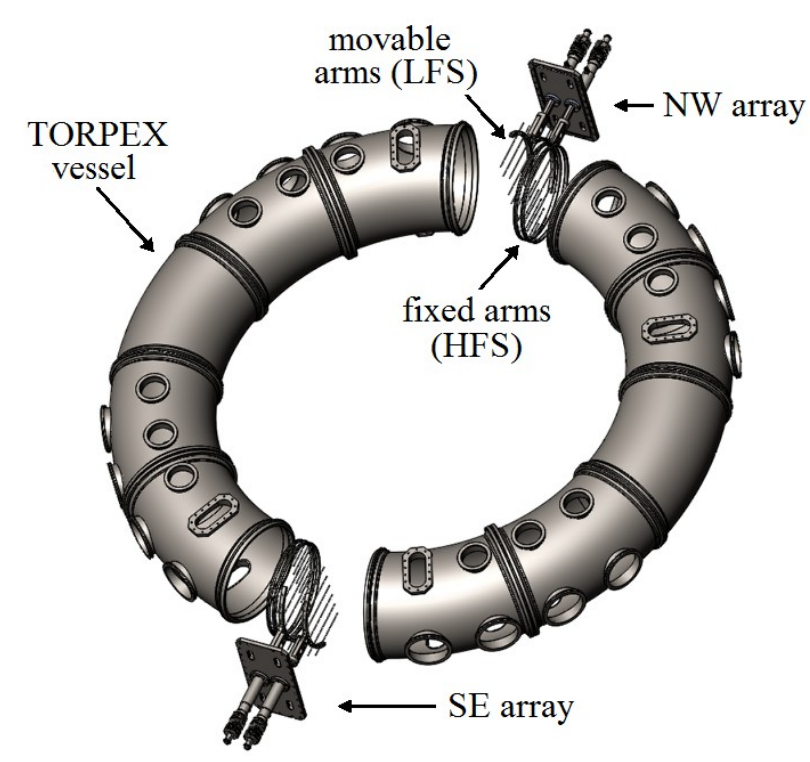

FIG. 4. Two complete LP arrays are installed on opposite toroidal locations in TORPEX, on the southeast (SE) and northwest (NW) areas. The movable LFS arms are installed separately from the fixed HFS arms and therefore have a small toroidal separation $\left(<10^{\circ}\right)$ from the latter. Each array has its own independent front-end electronics and data acquisition system.

sumed to reach the probe only from a direction parallel (or anti-parallel) to the magnetic field and not undergo collisions once they enter the presheath. If the probe is in ion-saturation mode, the current becomes proportional to $n$ according to the relation

$$
I_{\mathrm{ion}}^{\mathrm{sat}} \approx 0.5 q A_{\mathrm{eff}} n \sqrt{q T_{e} / m_{\mathrm{ion}}}
$$

Here $q$ is the elementary charge, $m_{\text {ion }}$ is the mass of the ions, $T_{e}$ is in electron-volts and $A_{\text {eff }}$ is the effective sheath area around the probe. $A_{\text {eff }}$ can be approximated ${ }^{2}$ by the value of the probe surface projected in the direction of the magnetic field. For the cylindrical shape of the HEXTIP-U probes, we make a projection perpendicular to the axis and obtain $A_{\text {eff }} \approx 2 \times 4 \mathrm{~mm} \times 2 \mathrm{~mm} \approx 16 \mathrm{~mm}^{2}$.

The ion-saturation mode is obtained by biasing the probe at a sufficiently negative voltage such that no electrons are collected. In practice, this means that further decreasing the voltage does not lead to an appreciable decrease in probe current, except for a small correction due to sheath expansion ${ }^{2}$. This condition is fulfilled in our setup at biases $\lesssim-20 \mathrm{~V}$. Then, using $T_{e}=5 \mathrm{eV}$, we can estimate an $I_{\text {ion }}^{\text {sat }} \approx\left(3 \times 10^{-20} \mathrm{~A} \mathrm{~m}^{3}\right) \times n \leq 3 \mathrm{~mA}$ for $\mathrm{H}$ plasmas with $n \leq 10^{17} \mathrm{~m}^{-3}$. It is important to note that Eq. 1 relies on several simplifications, so its use has significant uncertainty. It nevertheless gives us means to calculate approximate values of $I_{\text {ion }}^{\text {sat }}$ expected in our experiments and, conversely, to have a rough interpretation of $I_{\text {ion }}^{\text {sat }}$ measurements in terms of $n$, assuming 
fixed $T_{e}$. The value $T_{e}=5 \mathrm{eV}$ is consistent with measurements made using a more precise triple-probe ${ }^{21}$ in a similar setup. The triple-probe results, however, show that $T_{e}$ can vary across the poloidal cross-section, so care must be taken not to overextend the assumption of constant temperature. For the work presented here we are only concerned with $I_{\text {ion }}^{\text {sat }}$, so knowledge of the detailed profile of $T_{e}$ is not required.

$V_{p}$ can be determined from the zero-current probe potential (the floating potential, $V_{\mathrm{fl}}$ ) through

$$
V_{p}=V_{\mathrm{fl}}+\mu T_{e}
$$

where $T_{e}$ is in electron-volts and $\mu$ is a parameter that depends on the choice of gas and whose value was determined experimentally in TORPEX for hydrogen plasmas in a similar setup ${ }^{21}$ to be $\mu=3.1 \pm 0.2$.

The electronics of each of the two arrays of HEXTIP-U consist of 12 independent boards, each one dedicated to 8 probes, which can be set remotely and independently from one another to measure probe current or floating potential (Fig. 5). In the latter case, the boards act as simple high input-impedance $(\approx 10 \mathrm{M} \Omega)$ amplifiers which output a voltage proportional to the probe potential under negligible probe current conditions. In the former, the cards bias the probes at one of seven different choices of biasing potential $(-54 \mathrm{~V},-42 \mathrm{~V},-30 \mathrm{~V},-15 \mathrm{~V}$ or any one of three arbitrary voltages applied externally) and convert the value of probe current into a voltage in a range well suited for digitization by one of two 96channel, 16-bit, D-tAcq ACQ196CPCI data acquisition systems $^{23}$. The probe current circuitry includes a lowpass filtering stage for better noise immunity and to help prevent aliasing at the $250 \mathrm{kHz}$ sampling frequency ${ }^{24}$.

We performed a careful calibration of all channels in the two main operating modes ( $V_{\mathrm{fl}}$ and probe current) in order to have an accurate interpretation of the digitized signals as currents or floating potentials. We used linear transfer-function models as expected from the known circuit design and behavior of the data-acquisition system. Calculation of the model parameters using nominal component values from the circuit schematics gave significant errors (sometimes as high as $\sim 10 \%$ ) found to be caused mainly by component tolerances and, possibly, small component nonlinearities. Therefore, a more practical approach was followed where the parameters were estimated using least-squares fits of digitized signals resulting from known applied DC currents or low frequency voltages. This method gives much better results, as a detailed examination of the fits reveals. The $V_{\mathrm{fl}}$ mode shows average residuals (in absolute value) $<2 \mathrm{mV}$ in all channels for inputs in the range $-50 \mathrm{~V}$ to $30 \mathrm{~V}$. Similarly, for probe currents, probe-biases in the range $-54 \mathrm{~V}$ to $0 \mathrm{~V}$ show fit residuals $<2 \mu \mathrm{A}$ in all channels for input currents in the range $0-4 \mathrm{~mA}$. If we use Eq. 1 to get an idea of what these results mean in terms of densities, then the calibration introduces errors of approximately

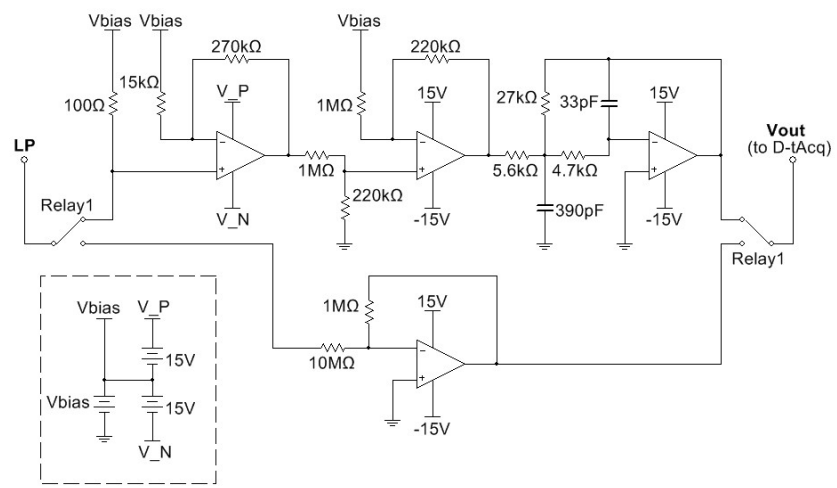

FIG. 5. Circuit schematics of the electronics for a single probe. The upper branch biases the probe (LP, on the left) at a voltage $\mathrm{V}_{\text {bias }}$ and measures the probe current. The lower branch measures the LP potential for negligible current. In both cases, the output is a voltage ( $\mathrm{V}_{\text {out }}$, on the right) well suited for digitization by the D-tAcq. The measurement mode (probe current or floating potential) is selected via a relay at the input and output as shown. The operational amplifier on the upper left uses the biasing arrangement shown inside the dashed-line box (lower left).

$\lesssim 7 \times 10^{13} \mathrm{~m}^{-3}$. This is a small number compared to typical TORPEX densities (see Sec. II).

The AC response of the probe current circuitry is tested by applying a current frequency sweep and comparing its constant amplitude to the amplitude of the calibrated (digitized) output signal at different values of frequency (Fig. 6). We observe the expected lowfrequency gain and low-pass behavior, although with $-3 \mathrm{~dB}$ cut-off frequencies slightly lower $(\approx 110 \mathrm{kHz})$ than the design value of half the D-tAcq sampling frequency $(250 \mathrm{kHz} / 2=125 \mathrm{kHz})$. A slight cross-talk at frequencies $>10 \mathrm{kHz}$, peaking at $\approx-40 \mathrm{~dB}$ near $100 \mathrm{kHz}$, is observed in non-excited channels located in very close proximity to an excited one. This value is negligible for most practical applications. The total measured rootmean-square (RMS) noise is $\leq 4 \mu \mathrm{A}_{\mathrm{RMS}}$ in all channels. This number includes possible noise contributions from the extra circuitry feeding the test current, estimated at $\leq 1 \mu \mathrm{A}_{\mathrm{RMS}}$, so the actual noise figure should be lower. For a range $-5 \mathrm{~mA}$ to $5 \mathrm{~mA}$ we can conclude that the corresponding noise level in terms of density (using Eq. 1) is $\lesssim 1.3 \times 10^{14} \mathrm{~m}^{-3}$. This noise figure sets the precision limit of the system, as other uncertainties like calibration errors, cross-talk (for channels not in close proximity or frequencies far from $100 \mathrm{kHz}$ ), and D-tAcq quantization $(\approx 0.15 \mu \mathrm{A}$ per step) are smaller.

In the case of floating potential, the circuitry is much simpler and less immune to noise. We measure typical noise voltages of $\approx 30 \mathrm{mV}$ RMS, although some channels are noisier $\left(\approx 100 \mathrm{mV}_{\mathrm{RMS}}\right)$. This does not constitute a serious shortcoming for our experiments, as we typically track potential variations in the order of $\sim 1 \mathrm{~V}$.

The possibility of using external biases allows for 
a)

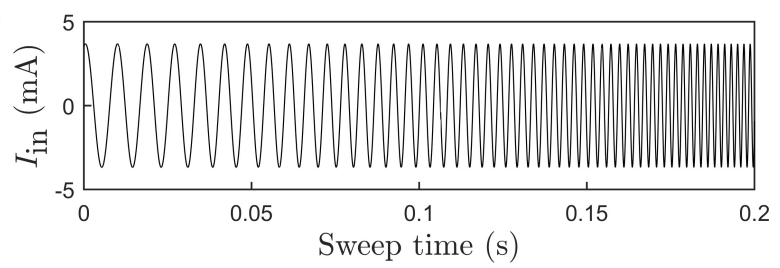

b)

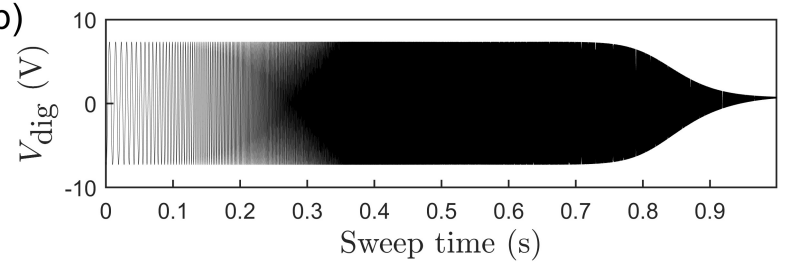

c)

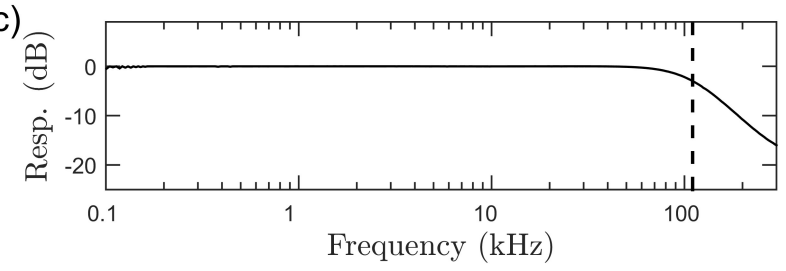

FIG. 6. Example of AC response of probe current circuitry. a) A current sinusoidal signal $I_{\text {in }}$, with constant amplitude and frequencies sweeping logarithmically from $100 \mathrm{~Hz}$ to $500 \mathrm{kHz}$ in $1 \mathrm{~s}$, is injected into channel 13 of the SE array (only the first $0.2 \mathrm{~s}$ are shown for clarity). b) A digitized voltage signal, $V_{\text {dig }}$ is then recorded at the output of the system which decreases in amplitude at high frequencies. This behavior is more clearly seen in c), where the amplitude of $V_{\text {dig, nor- }}$ malized to a maximum value of 1 and expressed in $\mathrm{dB}$, is plotted against sweep frequency. The $-3 \mathrm{~dB}$ level is reached at $\approx 110 \mathrm{kHz}$ (vertical dashed line).

voltage-sweep measurements ${ }^{21,25}$. By applying a sawtooth voltage bias while simultaneously measuring the probe currents, one can record complete I-V characteristics of the LPs and obtain experimental values of $T_{e}$, $V_{p}$ and $n$ from fits of ideal LP I-V curves to the data. Ideally, the sawtooth signal should have a high enough frequency for the plasma parameters not to change appreciably during one cycle. In practice, this would require bias frequencies and data acquisition rates too high to attain with our hardware. So, alternatively, we could obtain time-average values of $T_{e}, V_{p}$ and $n$ by using lower sweep frequencies $\sim 100 \mathrm{~Hz}$ and performing the fits on average I-V curves. This method has been used in previous studies with good results ${ }^{21}$. Nevertheless, difficulties may arise from biasing the probe arrays above floating potential, close to electron saturation $I_{\mathrm{e}}^{\text {sat }}$. In that regime, probe currents are expected to be much higher than at ion saturation ${ }^{2}$. For example, $\left|I_{\mathrm{e}}^{\text {sat }}\right| \approx 33 \times\left|I_{\mathrm{ion}}^{\text {sat }}\right|$ in hydrogen plasmas. This value poses a risk of adversely affecting the plasma, damaging the probes or overloading the electronics. For the present work, we are interested in fast changes of plasma structures, so we concentrate on $I_{\text {ion }}^{\text {sat }}$ and leave studies of time-average $n, T_{e}$ and $V_{p}$ profiles for future investigations.

We note that we do not expect the LP arrays to significantly affect our plasmas during $I_{\text {ion }}^{\text {sat }}$ measurements, as detailed below. Firstly, data taken in past experiments using a single, smaller, array ${ }^{19}$ showed similar values of $I_{\text {ion }}^{\text {sat }}$ for similar plasma configurations and a similar overall behavior, including the statistical properties of $I_{\mathrm{ion}}^{\text {sat }}$ fluctuations and of turbulent structures. As the number of LPs has now more than doubled, this observation is an indication that the arrays have no significant effects. Secondly, we use an argument based on the plasma confinement time $t_{c}$. Experiments performed with hydrogen in a similar setup ${ }^{26}$ gave $t_{c} \approx 0.2 \mathrm{~ms}$. This means that heating must continuously compensate for $n_{\text {typ }} V_{\text {ves }} / t_{c}$ particles being lost, with $n_{\text {typ }} \approx 10^{16} \mathrm{~m}^{-3}$ (a typical density) and $V_{\text {ves }}=0.8 \mathrm{~m}^{3}$ (the volume of the vessel). Therefore $n_{\mathrm{typ}} V_{\text {ves }} / t_{c} \approx 4 \times 10^{19} \mathrm{~s}^{-1}$. On the other hand, one typical probe measures on average $I_{\text {ion }}^{\text {sat }} \approx 0.1 \mathrm{~mA}$ (see Sec. IV). The total current drawn by the two LP arrays is then $190 \times I_{\mathrm{ion}}^{\mathrm{sat}}$, which in terms of particle flux is $\frac{d N_{\mathrm{p}}}{d t} \approx 190 I_{\mathrm{ion}}^{\mathrm{sat}} / q \approx 1.2 \times 10^{17} \mathrm{~s}^{-1}$ ( $q$ is the elementary charge). Thus $\frac{d N_{\mathrm{p}}}{d t} \ll n_{\text {typ }} V_{\text {ves }} / t_{c}$, so the fraction of particles that are collected by the arrays is much smaller than the number of particles that are continuously replenished and form the plasma bulk. The perturbation of HEXTIP-U on the plasmas is therefore expected to be unimportant.

We conclude this section by pointing out that the calibration and characterization of the electronics do not take into account any contributions from parts prior to the front-end. Actually, the only additional components are the cables and connectors leading to the probes (see Sec. II) which are not expected to have a significant effect. Associated parasitic capacitances would need to be better characterized in future non-standard modes of operation.

\section{TESTS AND ANALYSIS}

Previous experiments ${ }^{4,22,27,28}$ and simulations ${ }^{29}$ have shown that, for certain SMT configurations, TORPEX plasmas are characterized by the presence of an ideal interchange mode which generates intermittent fieldaligned plasma structures that propagate radially outward (the so-called blobs).

In the first set of tests, we choose one such magnetic configuration and measure coherences between points on two different toroidal locations of TORPEX using the probes on the NW and SE arrays of HEXTIP-U (see Fig. 4). We use hydrogen and $P_{\mathrm{mw}}=600 \mathrm{~W}$. The toroidal coils are energized at a current $I_{\phi}=360 \mathrm{~A}$, for an on-axis toroidal field $B_{\phi}=72.6 \mathrm{mT}$, and a vertical coil current is set in the range $I_{z}=55 \mathrm{~A}$ to $I_{z}=100 \mathrm{~A}$, for $B_{z}=1.2-2.1 \mathrm{mT}$. The $\mathrm{TC}$ is set to its maximum vertical position (removed from the plasma) and left deenergized. The calculated number of field turns around the toroidal vessel (from bottom wall to top wall, at 

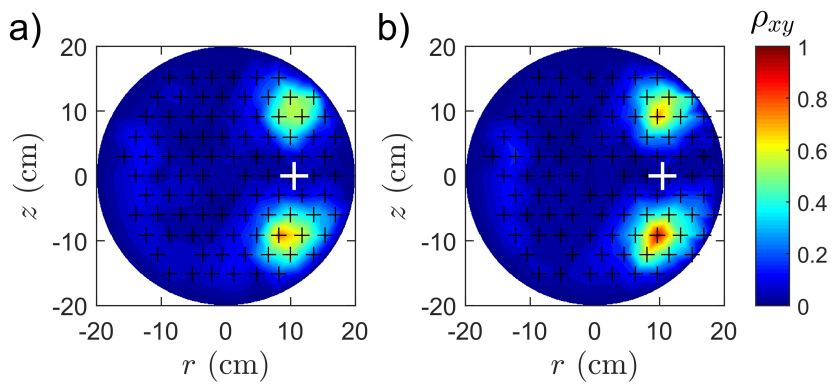

FIG. 7. Cross-correlation $\rho_{x y}$ (see text for definition) between NW probe 21 (white cross) and all SE array probes (black crosses) for $I_{z}=77 \mathrm{~A}$ and different values of LFS probe radial displacement $\Delta_{r}$ : (a) $\Delta_{r}=-5 \mathrm{~mm}$ (TORPEX experimental shot 67391) and (b) $\Delta_{r}=+10 \mathrm{~mm}$ (shot 67372). Note that all black crosses are at approximately the same toroidal location while the white cross is separated $180^{\circ} . \rho_{x y}$ values range from -1 to 1 and give zero when signals are uncorrelated; however, the colormap only spans 0 to 1 as no negative $\rho_{x y}$ were observed. Data is interpolated linearly throughout the poloidal cross section assuming zero correlation between NW probe 21 and the vessel wall. We estimate errors $\delta\left(\rho_{x y}\right) \leq$ 0.012 in all cases using studies of the variation of $\rho_{x y}$ with subsets of the data of length $500 \mathrm{~ms} . \Delta_{r}=+10 \mathrm{~mm}$ is the displacement that gives the largest correlation of NW probe 21 with any SE probe. In that case, $\rho_{x y}=0.906 \pm 0.002$ with SE probe 29 .

$r=0)$ for these parameter values is $N=2.2$ to $N=3.8$.

All HEXTIP-U probes are biased at $-42 \mathrm{~V}$ and $I_{\text {ion }}^{\text {sat }}$ data is collected in different trials of duration $1.0 \mathrm{~s}$. The NW array is set to zero LFS arm displacement and kept fixed in all runs. The LFS arms of the other array $(\mathrm{SE})$ are displaced between runs in steps of $5 \mathrm{~mm}$ in the $r$ direction by means of the linear-motion actuator (Sec. II). We compute, for each trial, the normalized cross-correlation ${ }^{30-32}$ of pairs of time-series $\langle x, y\rangle$ (where $x$ corresponds to a NW-array probe and $y$ to a SE probe), for a range of time-lags, to allow for the possibility that the same plasma structure reach the two probes at slightly different times due to possible finite structure propagation speeds. The result is a function of correlation vs time-lag which exhibits a maximum value $\rho_{x y}$. Figure 7 shows the results obtained by comparing values of $\rho_{x y}$ for different array shifts $\left(\Delta_{r}\right)$. These results are consistent with the expectation that higher correlations should be observed between probes lying near the same magnetic field line.

Changes in $B_{z}$ produce changes in $N$, so it is expected that $\rho_{x y}$ also vary with changes in $I_{z}$. Figure 8 shows cross-correlations between NW probe 21 and SE probe 29 for a parameter scan in $I_{z}$ and $\Delta_{r}$. These probes lie in a region of strong plasma intermittence ${ }^{22}$ and, from the results above, are expected to be located near the same magnetic field line. The existence of a coherence maximum, as well as the observation that the average time-lag at the maximum is $\approx 0 \mu \mathrm{s}$, are an indication of
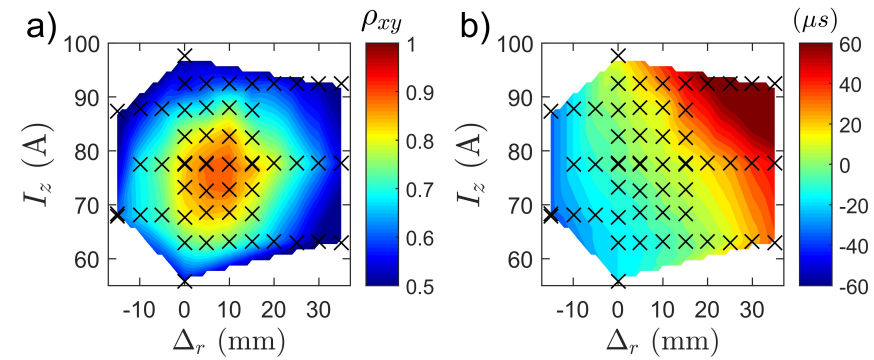

FIG. 8. (a) Cross-correlation $\rho_{x y}$ between NW probe 21 and SE probe 29 for different values of $I_{z}$ and $\Delta_{r}$. SE probe 29 gives the largest $\rho_{x y}$ between any SE array probe and NW probe 21 . The black X-markers indicate the choice of $I_{z}$ and $\Delta_{r}$ for each measurement; data is linearly interpolated for other points. Notice the colormap range 0.5 to 1.0 , chosen for better contrast. In all measurements an error $\delta\left(\rho_{x y}\right) \leq 0.010$ is estimated the same way as in Fig. 7. However, the error is much smaller $(\leq 0.002)$ in measurements near the largest values of $\rho_{x y}$. (b) Time-lag of signals at which the maximum value of cross-correlation occurs.

an optimum alignment of the two probes made possible by virtue of the radial displacement control of the new diagnostic.

In the second set of tests, we use HEXTIP-U to image hydrogen plasma structures in an SMT configuration. We set $I_{\phi}=360 \mathrm{~A}, I_{z}=77 \mathrm{~A}, P_{\mathrm{mw}}=600 \mathrm{~W}$ and, for each acquisition time step, obtain 2D poloidal images of $I_{\text {ion }}^{\text {sat }}$ for the two LP arrays by interpolating $I_{\text {ion }}^{\text {sat }}$ probe data over each poloidal cross-section, assuming $I_{\text {ion }}^{\text {sat }}=0$ at the wall (see Fig. 9). We then single-out "raw" shapes within the images by finding all disjoint sets of image pixels for which $I_{\mathrm{ion}}^{\text {sat }} \geq 0.16 \mathrm{~mA}$ (corresponding to $n \approx 10^{16} \mathrm{~m}^{-3}$ from Eq. 1). Then, using a simplified model of the magnetic field, we identify 3D plasma structures by determining the shapes that are linked by magnetic field lines on the same or opposite LP arrays. By repeating this procedure in successive time frames and properly combining results, we can track the time evolution of the structures and their basic 3D features. Figure 10 shows an example of typical results obtained using this methodology.

Lastly, we position the TC at the center of the poloidal plane $(r=0, z=0)$. By setting $I_{\phi}=390 \mathrm{~A}, I_{z}=19 \mathrm{~A}$ and energizing the TC with $I_{\mathrm{TC}}=710 \mathrm{~A}$, we create a magnetic configuration with mostly closed field lines and a small region of open field lines near the LFS (see Fig. 11a). Using hydrogen and $P_{\mathrm{mw}}=800 \mathrm{~W}$, we follow the same methodolgy above to identify and track the evolution of 3D plasma structures, setting a threshold $I_{\text {ion }}^{\text {sat }} \geq 0.80 \mathrm{~mA}$ (approximately $n \approx 5 \times 10^{16} \mathrm{~m}^{-3}$ from Eq. 1) to determine separate 2D shapes. Figure 11 shows four consecutive frames of evolving plasma structures. The apparent changes in the plasma, compared to Figs. 9, 10, are partly due to the change in $I_{\phi}$ and the consequent location of the electron-cyclotron and upper- 

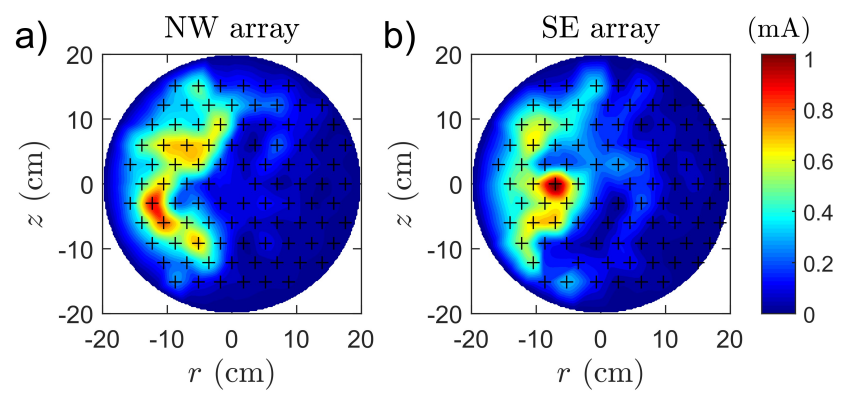

FIG. 9. Instantaneous interpolated $I_{\text {ion }}^{\text {sat }}$ profile as seen on the (a) NW and (b) SE LP arrays. From Eq. 1, $I_{\text {ion }}^{\text {sat }}$ can be used as a proxy for the density $n$ assuming constant $T_{e}$. The ' + ' signs mark the location of the LPs, as illustrated in Fig. 1a. The data presented here corresponds to one particular time frame of shot 67372 .
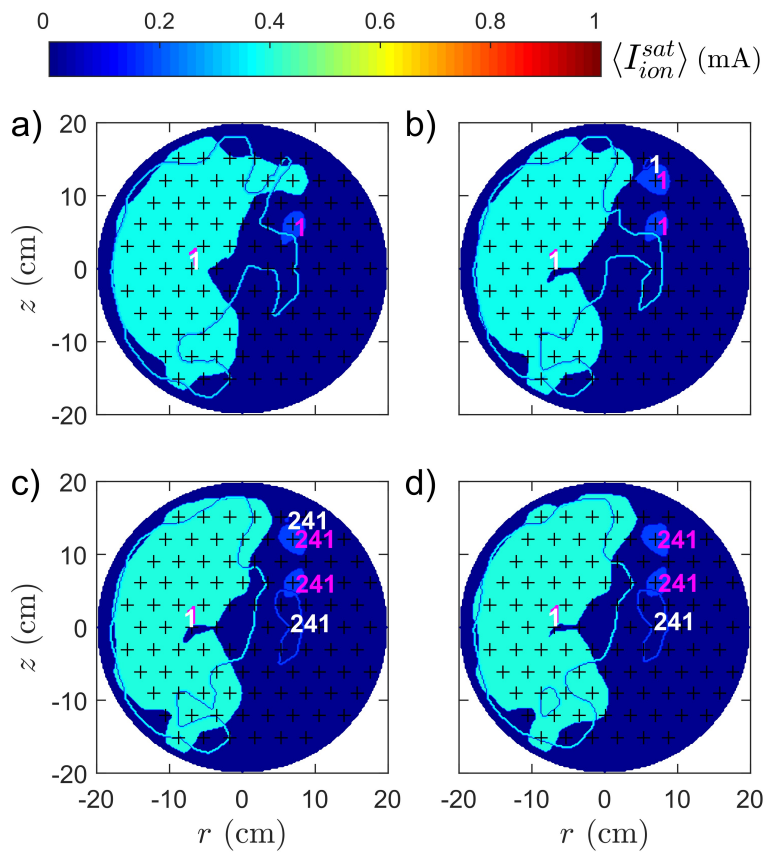

FIG. 10. Plasma structures identified with HEXTIP-U in an SMT run. Solid shapes are NW-array data and hollow shapes come from the SE array. The latter are boundaries of otherwise solid shapes; the interior is left blank only to superpose information from both arrays on the same plot. Colors indicate the average ion saturation current of all points within the shape $\left\langle I_{\text {ion }}^{\text {sat }}\right\rangle$ as per the colorbar. Numbers on or next to shapes (white for SE-array, magenta for NW-array) indicate the number of the 3D-structure that the shapes make part of. These structure numbers are assigned consecutively from the start of the shot. The ' + ' signs mark the location of NW-array probes. (a-d) Successive time frames, in steps of $4 \mu \mathrm{s}$, that show the evolution of $3 \mathrm{D}$ plasma structures. Plot (a) corresponds to the same plasma run and time as Fig. 9. Structure 1 is identified as the main plasma. Structure 241 forms upon separation from structure 1 .
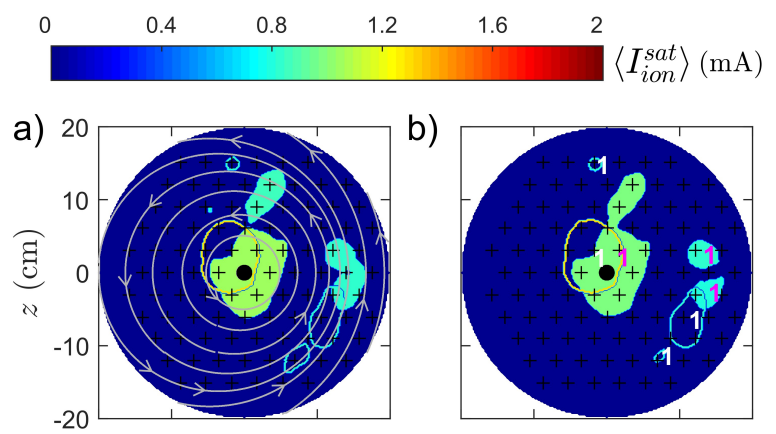

b)
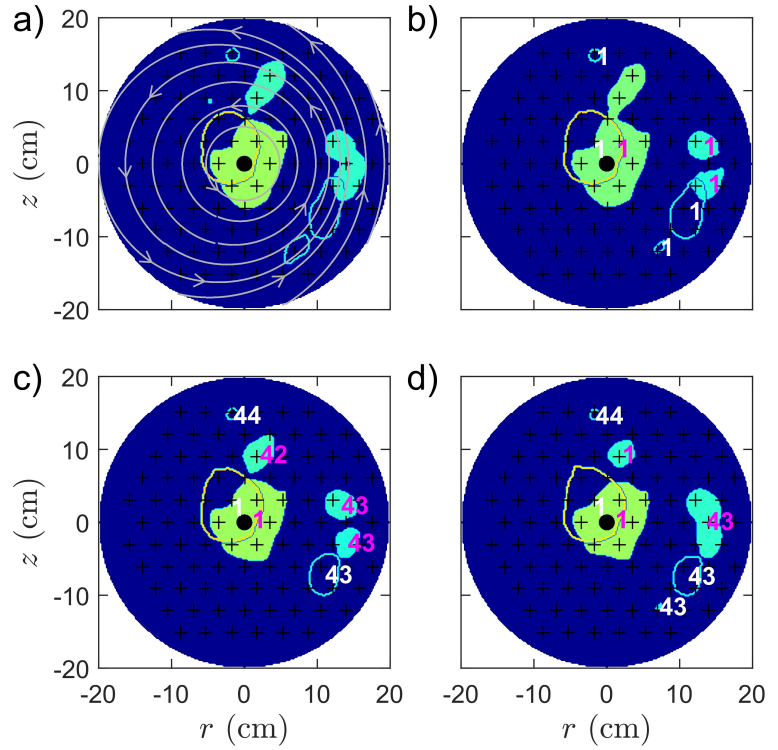

FIG. 11. Plasma structures identified with HEXTIP-U in a closed magnetic field line configuration. We use the same methodology for structure identification and plotting as in Fig. 10. Notice the change in the colormap which corresponds to the detection of structures with higher average ion saturation current $\left\langle I_{\mathrm{ion}}^{\mathrm{sat}}\right\rangle$. The black dot at $z=0, r=0$ shows the location of the TC. (a-d) Evolution of plasma structures in successive time-frames. The gray arrows in (a) show the calculated magnetic field lines projected on the poloidal plane; no structure number labels are shown there to avoid image cluttering.

hybrid resonance layers ${ }^{13}$; but, more interestingly, they are the result of the change in magnetic configuration. More complete studies of the evolution of the plasma with closed field lines in TORPEX will be performed in the future.

\section{CONCLUSIONS}

In this paper, we describe the construction, installation and commissioning of HEXTIP-U, a new diagnostic based on two LP arrays that gives TORPEX new capabilities for studies on the dynamics of plasma structures.

The new system allows us to determine basic 3D features of the plasmas, as established with results from three different experiments. In the first one, we study the coherence between LPs on toroidally-opposite sides of the TORPEX apparatus using an SMT plasma. We obtain high correlations for probes lying near the same magnetic field lines, an observation that supports the expectation that plasma structures are elongated along magnetic field lines. This study is performed in detail thanks to the ability to fine-tune the $r$ location of LFS probes by virtue of 
the linear-motion actuator of HEXTIP-U.

The second experiment shows an example of the observation of a plasma as it evolves in time, taking into account basic 3D features. Analysis routines are developed to detect 2D shapes on the two LP arrays and then reconstruct 3D plasma structures by forming groups of shapes linked by magnetic field lines. In this way, we combine data from distinct LP arrays to gain more complete information on the plasmas. We can now, for example, study structure changes in the direction parallel to the magnetic field, a functionality that may be of importance for future studies on coherent structures and the 3D structure and dynamics of plasma blobs.

The linear-motion actuator makes comprehensive poloidal cross-section coverage of HEXTIP-U compatible with TC operations. The usefulness of such a feature was demonstrated in the third set of experiments, where the evolution of plasmas was observed in a closed magnetic field line configuration. Further dedicated studies will follow to fully explore plasma dynamics with closed field lines, a subject that is of direct relevance to the understanding of Tokamak physics.

\section{ACKNOWLEDGMENT}

The authors wish to acknowledge the support of the Swiss Plasma Center technical team.

This work is partly funded by the Swiss National Science Foundation.

${ }^{1}$ V.I. Demidov, S.V. Ratynskaia and K. Rypdal, Electric probes for plasmas: The link between theory and instrument, Rev. Sci. Instrum. 73, 3409 (2002).

${ }^{2}$ I.H. Hutchinson, Principles of plasma diagnostics, Cambridge University Press, Cambridge, 1987.

${ }^{3}$ I. Furno, F. Avino, A. Bovet, A. Diallo, A. Fasoli, K. Gustafson, D. Iraji, B. Labit, J. Loizu, Müller, G. Plyushchev, M. Podesta, F.M. Poli, P. Ricci and C. Theiler, Plasma turbulence, suprathermal ion dynamics and code validation on the basic plasma physics device TORPEX, J. Plasma Phys. 81, 345810301 (2015).

${ }^{4}$ I. Furno, B. Labit, M. Podesta, A. Fasoli, S.H. Müller, F.M. Poli, P. Ricci, C. Theiler, S. Brunner, A. Diallo and J. Graves, Experimental observation of the blob-generation mechanism from interchange waves in a plasma, Phys. Rev. Lett. 100, 055004 (2008).

${ }^{5}$ S.H. Müller, C. Theiler, A. Fasoli, I. Furno, B. Labit, G.R. Tynan, M. Xu, Z. Yan and J.H. Yu, Studies of blob formation, propagation and transport mechanisms in basic experimental plasmas (TORPEX and CSDX), Plasma Phys. Control. Fusion 51, 055020 (2009).

${ }^{6}$ N. Katz, J. Egedal, W. Fox, A. Le and M. Porkolab, Experiments on the propagation of plasma filaments, Phys. Rev. Lett. 101, 015003 (2008).

${ }^{7}$ M.J. Burin, G.R. Tynan, G.Y. Antar, N.A. Crocker and C. Holland, On the transition to drift turbulence in a magnetized plasma column, Phys. Plasmas 12, 052320 (2005).

${ }^{8}$ M. Gilmore, W.A, Peebles and X.V. Nguyen, Detailed comparison of plasma turbulence correlation length measurements using microwave reflectometry and a Langmuir probe array, Plasma Phys. Control. Fusion 42, L1-L7 (2000).

${ }^{9}$ D.A. D'Ippolito, J.R. Myra and S.J. Zweben, Convective transport by intermittent blob-filaments: Comparison of theory and experiment, Phys. Plasmas 18, 060501 (2011).
${ }^{10}$ F.M. Poli, M. Podesta and A. Fasoli, A robust method for measurement of fluctuation parallel wavenumber in laboratory plasmas, Rev. Sci. Instrum. 80, 053501 (2009).

${ }^{11} \mathrm{~J}$. Angus, M. Umansky and S. Krasheninnikov, Effect of drift waves on plasma blob dynamics, Phys. Rev. Lett. 108, 215002 (2012).

${ }^{12}$ A. Fasoli, A. Burckel, L. Federspiel, I. Furno, K. Gustafson, D. Iraji, B. Labit, J. Loizu, G. Plyushchev, P. Ricci, C. Theiler, A. Diallo, S.H. Müller, M. Podesta and F. Poli, Electrostatic instabilities, turbulence and fast ion interactions in the TORPEX device, Plasma Phys. Control. Fusion 52, 124020 (2010).

${ }^{13}$ M. Podesta, A. Fasoli, B. Labit, M. McGrath, S.H. Müller and F.M. Poli, Plasma production by low-field side injection of electron cyclotron waves in a simple magnetized torus, Plasma Phys. Control. Fusion 47, 19892002 (2005).

${ }^{14} \mathrm{~F}$. Avino, A. Fasoli and I. Furno, The new TORPEX in-vessel toroidal conductor for the generation of a poloidal magnetic field, Rev. Sci. Instrum. 85, 033506 (2014).

${ }^{15} \mathrm{~F}$. Avino, Turbulence at the boundary of toroidal plasmas with open and closed magnetic flux surfaces, Ph.D. dissertation (Ecole Polytechnique Fédérale de Lausanne, Switzerland, 2015).

${ }^{16}$ F. Avino, A. Fasoli, I. Furno, S. Jolliet and P. Ricci, Basic characterization of TORPEX electrostatic modes in closed field line configurations, Phys. Plasmas 21, 122115 (2014).

${ }^{17}$ F. Avino, A. Fasoli, I. Furno, P. Ricci and C. Theiler, X-point effect on plasma blob dynamics, Phys. Rev. Lett. 116, 105001 (2016).

${ }^{18}$ F. Piras, S. Coda, I. Furno, J.M. Moret, R.A. Pitts, O. Sauter, B. Tal, G. Turri, A. Bencze, B.P. Duval, F. Felici, A. Pochelon and C. Zucca, Snowflake divertor plasmas on TCV, Plasma Phys. Control. Fusion 51, 055009 (2009).

${ }^{19}$ S.H. Müller, A. Fasoli, B. Labit, M. McGrath, O. Pisaturo, G. Plyushchev, M. Podesta and F.M. Poli, Basic turbulence studies on TORPEX and challenges in the theory-experiment comparison, Phys. Plasmas 12, 090906 (2005).

${ }^{20}$ P.M. Bellan, Fundamentals of plasma physics, Cambridge University Press, Cambridge, 2006.

${ }^{21}$ C. Theiler, I. Furno, A. Kuenlin, Ph. Marmillod and A. Fasoli, Practical solutions for reliable triple probe measurements in magnetized plasmas, Rev. Sci. Instrum. 82, 013504 (2011).

${ }^{22}$ I. Furno, C. Theiler, V. Chabloz, A. Fasoli and J. Loizu, Presheath density drop induced by ion-neutral friction along plasma blobs and implications for blob velocities, Phys. Plasmas 21, 012305 (2014).

${ }^{23}$ See http://www.d-tacq.com/acq196cpci.shtml for ACQ196CPCI digitizer specifications.

${ }^{24}$ J.G. Proakis and D.G. Manolakis, Digital signal processing, 3rd ed. Prentice-Hall Inc, New Jersey, 1996, pp. 21-33.

${ }^{25}$ G. Chiodini, C. Riccardi and M. Fontanesi, A $400 \mathrm{kHz}$, fastsweep Langmuir probe for measuring plasma fluctuations, Rev. Sci. Instrum. 70, 2681 (1999).

${ }^{26}$ M. Podesta, A. Fasoli, B. Labit, M. McGrath, S.H. Müller and F.M. Poli, Experimental characterization and modelling of the particle source in an Electron-Cyclotron wave driven toroidal plasma, Plasma Phys. Control. Fusion 48, 1053-1062 (2006).

${ }^{27}$ F.M. Poli, P. Ricci, A. Fasoli and M. Podesta, Transition from drift to interchange instabilities in an open magnetic field line configuration, Phys. Plasmas 15, 032104 (2008).

${ }^{28}$ I. Furno, M. Spolaore, C. Theiler, N. Vianello, R. Cavazzana and A. Fasoli, Direct two-dimensional measurements of the fieldaligned current associated with plasma blobs, Phys. Rev. Lett. 106, 245001 (2011).

${ }^{29}$ P. Ricci, F.D. Halpern, S. Jolliet, J. Loizu, A. Mosetto, A. Fasoli, I. Furno and C. Theiler, Simulation of plasma turbulence in scrape-off layer conditions: the GBS code, simulation results and code validation, Plasma Phys. Control. Fusion 54, 124047 (2012).

${ }^{30} \mathrm{H}-\mathrm{J}$. Hartfuss and T. Geist, Fusion plasma diagnostics with $\mathrm{mm}$ waves, Wiley-VCH, Weinheim (Germany), 2013, pp. 265-268.

${ }^{31}$ J.E. Freund, Modern elementary statistics, 4th ed. Prentice-Hall 
Inc, New Jersey, 1973, pp. 420-447.

${ }^{32}$ P. Giudici, Applied data mining, John Wiley and Sons Ltd, West

Sussex (England), 2003, pp. 45-72. 